Title:

\title{
SPLIT-TARGET NEUTRONICS AND THE MLNSC SPALLATION TARGET SYSTEM
}

$$
\text { CONF-961110--19 }
$$

Author(s):

G. J. Russell, P. D. Ferguson, E. J. Pitcher, J. D. Court

\section{MASTÉa}

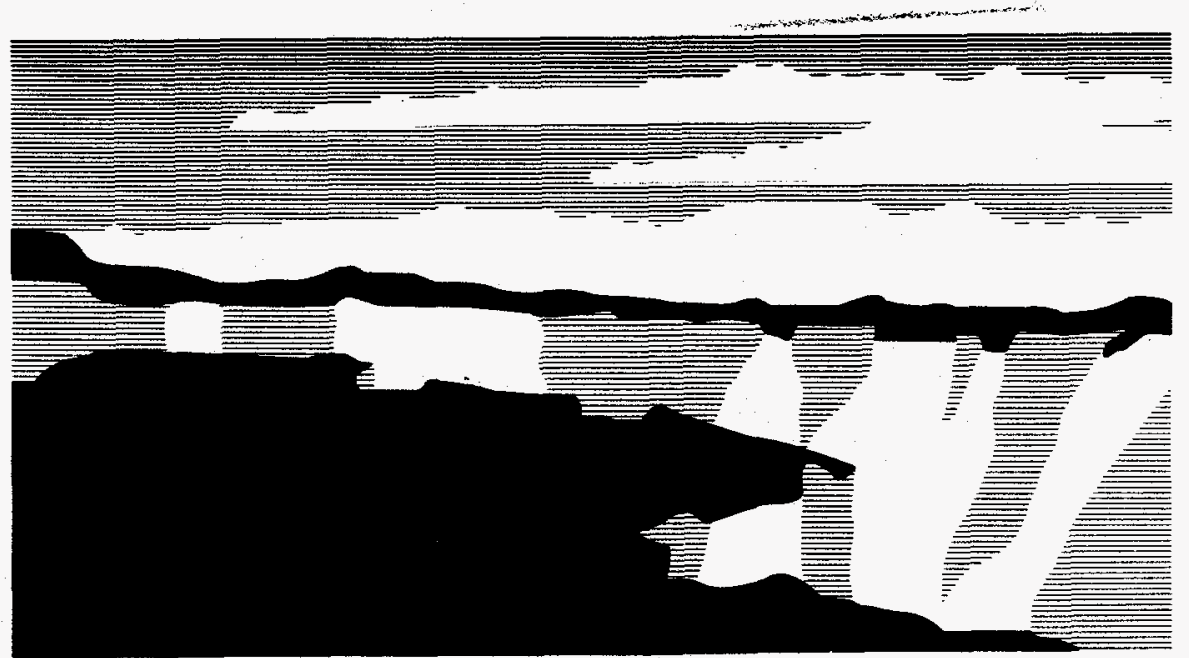

Los Alamos National Laboratory, an affirmative action/equal opportunity employer, is operated by the University of California for the U.S. Department of Energy under contract W-7405-ENG-36. By acceptance of this article, the publisher recognizes that the U.S. Government retains a nonexclusive, royalty-free license to publish or reproduce the published form of this contribution, or to allow others to do so, for U.S. Government purposes. The Los Alamos National Laboratory requests that the publisher identify this article as work performed under the auspices of the U.S. Department of Energy. 


\section{DISCLAIMER}

Portions of this document may be illegible in electronic image products. Images are produced from the best available original document. 


\title{
Split-Target Neutronics and the MLNSC Spallation Target System
}

\author{
G. J. Russell, P. D. Ferguson, E. J. Pitcher and J. D. Court \\ Manuel Lujan, Jr., Neutron Scattering Center, Los Alamos National Laboratory, Los Alamos, New Mexico 87545 \\ The Manuel Lujan, Jr., Neutron Scattering Center (MLNSC) at the Los Alamos National Laboratory is one of four \\ operating Short-Pulse Spallation Sources worldwide. The MLNSC target system (composed of targets, moderators, \\ and reflectors) was first installed in 1985 . The target system employs a split tungsten spallation target with a void \\ space in between (the flux-trap gap); this target system will be upgraded in 1998 . The ability to efficiently split a \\ spallation target allowed us to introduce the concept of flux-trap moderators and ultimately the notion of backscattering \\ and upstream moderators. The upgraded MLNSC target system will employ both flux-trap and \\ upstream/backscattering moderators to simultaneously service 16 neutron flight paths with high-intensity neutron \\ beams for materials science research.
}

\section{INTRODUCTION}

The Manuel Lujan Jr. Neutron Scattering Center (MLNSC) at the Los Alamos National Laboratory is one of four operating Short-Pulse Spallation Sources (SPSS) worldwide. Protons from the $800-\mathrm{MeV}$ Los Alamos Neutron Science Center accelerator (formerly Los Alamos Meson Physics Facility) impinge vertically downward onto the MLNSC spallation target system composed of spallation targets, moderators, and reflectors. ${ }^{1}$ The MLNSC target system(2), which was first installed in 1985, employs a split tungsten target with a void space in between (the fluxtrap gap); this target system will be upgraded in 1998. The ability to efficiently split a spallation target allowed us to introduce the concept of flux-trap moderators and ultimately the notion of backscattering and upstream moderators. Flux-trap moderators have several inherent neutronic advantages: a) all moderators are high-intensity; b) the neutron spatial distribution is fairly uniform over the moderator surface, and c) the moderators can be viewed in either transmission or backscattering geometry. We will discuss the rationale behind split targets, flux-trap and backscattering moderators, and the application of these concepts to the existing and upgraded MLNSC target s. stems.

Figure 1 shows the basic target-moderator geometries that have been (or will be) utilized in SPSS target systems. The most traditional geometry is a solid spallation target and wing moderators. The notion of a split spallation target was pioneered at Los Alamos, and is currently used in the MLNSC target system.

\section{SPLIT-TARGET NEUTRONICS}

An important general objective in the design of a spallation source target system is to maximize neutron production. Total neutron production (per incident particle) depends

\footnotetext{
${ }^{1}$ The basic notion of spallation and spallation targets is discussed in Ref. (1).
}

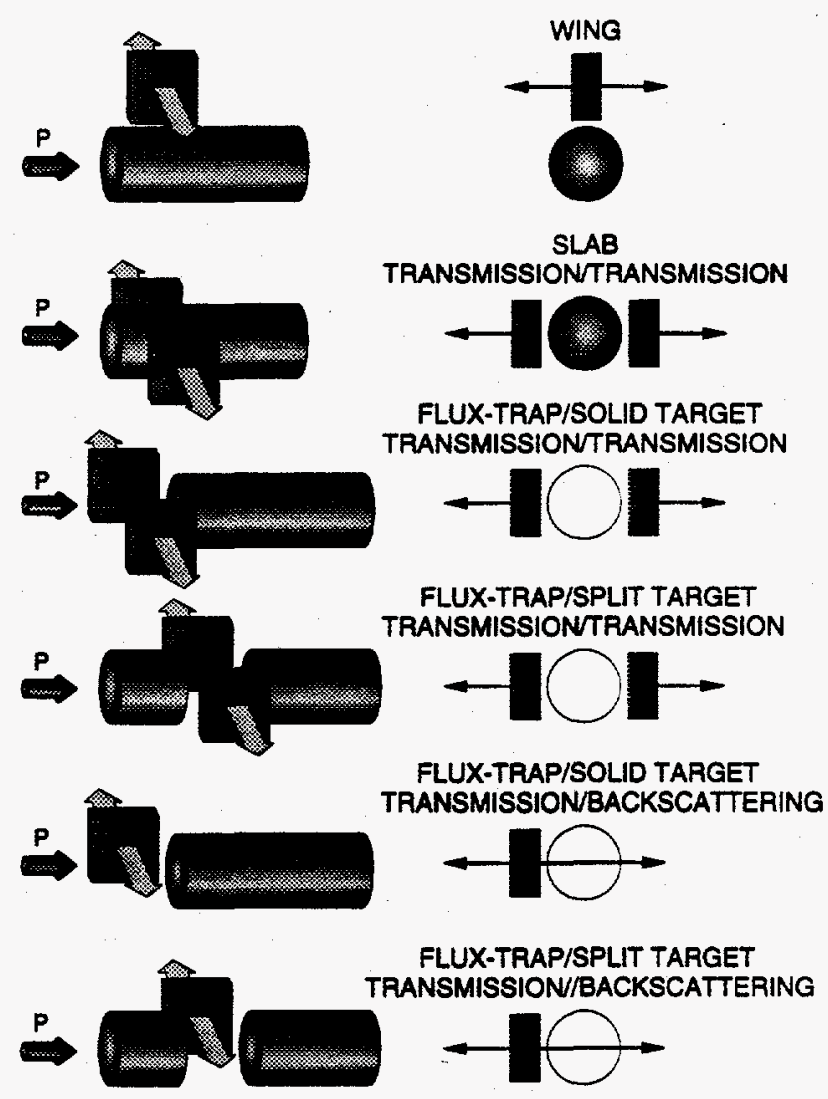

FIGURE. 1. Basic spallation target-moderator configurations.

essentially on the target material, the amount of material in the incident beam, geometry, and the energy and type of the incident particles. Two projectiles are generally considered for the incident beam: protons and deuterons; however, we confine our discussion here to protons. Once neutrons are produced inside the target, they must leak from the target before they can be used. Therefore, the other crucial aspect of spallation source target design is the maximization of the 
leakage of low-energy neutrons from the target. The main factors controlling neutron leakage are parasitic absorption in the target material and target geometry. The three materials of choice for practical neutron production (solid) targets are lead, tantalum, and tungsten. Depleted uranium has been used at the ISIS facility, and liquid mercury is under study as a target for the European Spallation Source (ESS) project.

Figure 2 shows the effect of splitting a tungsten spallation target: the neutron leakage for a split target is only about $10 \%$ less than a solid target with a target gap (flux trap) of $14 \mathrm{~cm}$ and a parabolic proton beam profile. Flux-trap gaps do not really affect the protons as they travel unhindered from target region to target region until a stopping length of target material finally halts their movement. Whether the stopping length of material is lumped in one solid piece or spread over a number of target segments is almost of no consequence for the primary protons.

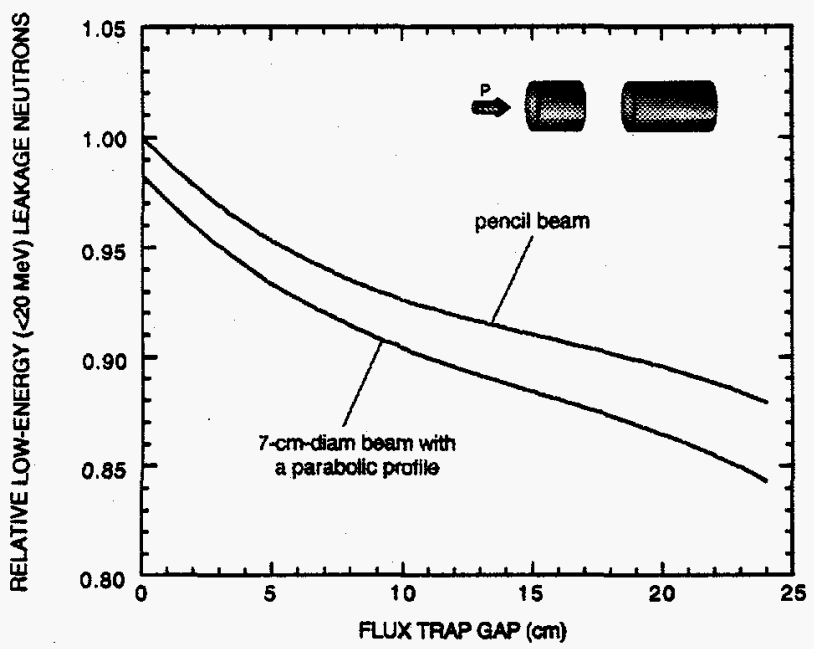

FIGURE 2. MLNSC split-target, flux-trap gap study.

The ability to efficiently split a target allowed us to introduce the concept of flux-trap moderators(3) and ultimately the concept of backscattering and upstream moderators(3). The relative performance of upstream, central, and downstream flux-trap moderators is illustrated in Fig. 3; the data shows the potential of "upstream" rivoderators compared to "downstream" moderators.

\section{POISONS, DECOUPLERS, AND LINERS}

For most users of a pulsed spallation-neutron source, useful neutrons can be defined as those headed in the right direction with appropriate energy at the right time. Unfortunately, spailation neutrons produced directly in the target rarely have the desired characteristics. We must, therefore, add the necessary systems and devices to the bare neutron production target in order to tailor the neutron pulse so that its characteristics are as close as possible to the users' requirements. As mentioned above, a complete target system consists not only of target(s) for the production of neutrons, but also of moderators, reflectors, and, in the case of an SPSS, poisons, decouplers, liners.

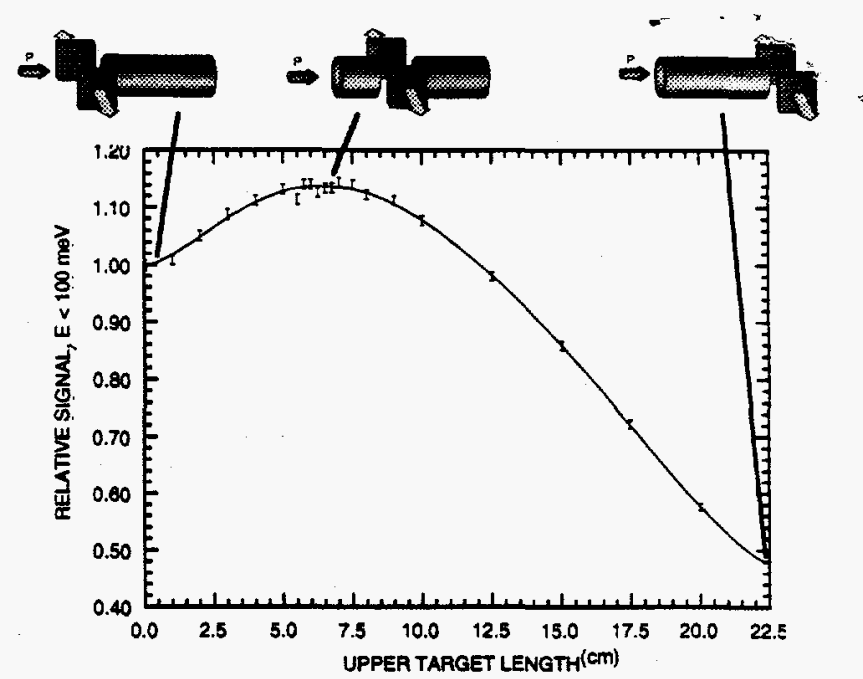

FIGURE 3. Illustration of upstream, central, and downstream flux-trap moderators viewed in transmission.

In addition to the choice of material, temperature, geometry (e.g., wing versus flux-trap moderators), and the presence or absence of a reflector, moderator neutronic performance is also strongly tied to the presence or absence of poisons, decouplers, and liners. The choice of materials and thickness for these target system components is a crucial part of moderator design(3).

The function of poisons, decouplers and liners is to tailor the temporal and energy characteristics of the neutron pulses emitted by the moderator(4). Figure 4 shows the arrangement of poisons, decouplers and liners in the splittarget, flux-trap moderator geometry.

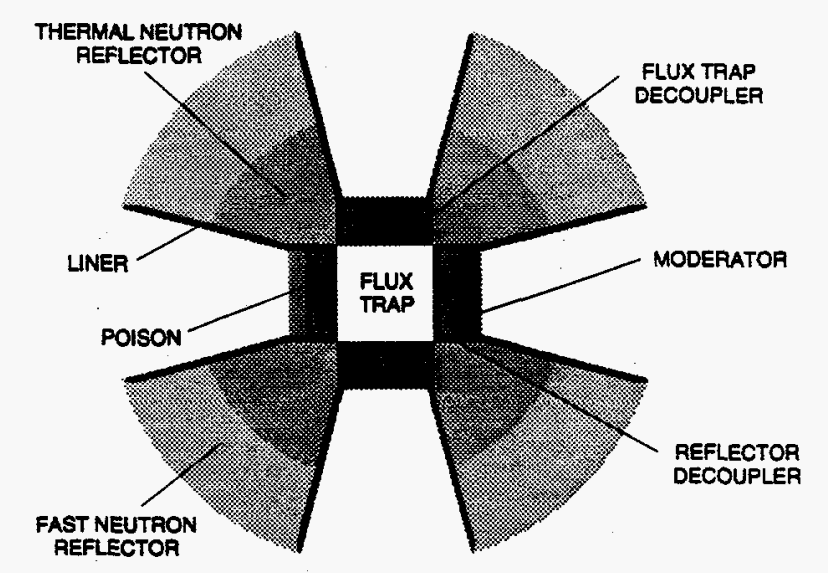

FIGURE 4. Arrangement of poisons, decouplers, and liners in a flux-trap moderator geometry. Poisons are typically oriented parallel to and positioned some distance $(=1$ to $3 \mathrm{~cm})$ behind the moderator viewed surfaces. The flux-trap decoupless neutronically insulate moderators from one another whereas the reflector decouplers neutronically isolate moderators from the adjoining reflector material. Liners neutronically insulate the reflector from the moderator viewed-surface.

For thermal neutrons, the poison neutronically defines that part of the moderator "viewed" by an experiment. Decouplers surround a moderator and both geometrically 
and neutronically isolate it from the reflector. Liners geometrically and neutronically isolate the moderator "viewed surface" from the reflector. The goal of short-pulse moderator design is to get as much useful neutron intensity from a moderator as possible with little or no attendant degradation in the neutron pulse width.

\section{CALCULATED RESULTS}

The time-averaged neutron source brightness from $5 \times 13 \times 13$ $\mathrm{cm}$ liquid hydrogen flux-trap moderators and composite reflectors is illustrated in Figs. $5 \mathrm{a}$ and $5 \mathrm{~b}$. The moderator geometry is depicted in Fig. 4; the overall reflector size was $114 \mathrm{~cm}$ diam. by $114 \mathrm{~cm}$ high. The proton energy was 800 $\mathrm{MeV}$, and the targets were stopping-length $(22.5 \mathrm{~cm})$, lightwater-cooled (pure) tungsten plates with a diameter of 10 $\mathrm{cm}$. The type and size of the inner reflector was varied in the calculations. The ortho/para-hydrogen mix was assumed to be $50 / 50 \mathrm{v \%}$. We show data for four composite reflectors: beryllium/lead, graphite/lead, light-water/lead, and heavy-water/lead (lead is always the outer reflector).

For a decoupled system with an inner graphite reflector, moderator performance is essentially independent of the size of the inner reflector. For a decoupled system and lightwater and heavy-water inner reflectors, moderator neutronic performance decreases when the inner reflector radius is increased. This is due to too much moderation occurring in the inner reflector with subsequent capture of neutrons in the decoupler/liner. materials. For an inner reflector of beryllium, the moderator performance continually increases with increased radius of the inner reflector. Note that asymptotic neutronic performance is reached for an inner reflector radius of $30-35 \mathrm{~cm}$.

For a coupled system with beryllium and liquid-deuterium reflectors, moderator performance increases as the size of the inner reflector becomes larger. Except for the very first calculated point at $15 \mathrm{~cm}$ radius, the moderator neutronic performance for an inner light water reflector of light water decreases with increasing inner reflector radius, reaching an asymptotic value at around $35 \mathrm{~cm}$ radius. The data point at $15 \mathrm{~cm}$ is interesting and indicates that neutronic gains may be made using light water as a premoderator for a liquid h $\gamma$ drogen moderator and a lead reflector. For a coupled system with an inner graphite reflector, moderator peiformance is essentially independent of the size of the inner reflector. For coupled moderators, the neutronic performance of the various composite reflectors reach asymptotic values at different inner reflector radii.

We show here only time-integrated data. Clearly, for shortpulse spallation source (decoupled systems) and long-pulse spallation source (coupled systems) applications, adequate time-dependent neutronic performance is imperative(5). We have time-dependent data for these calculations, but it is beyond the scope of this work to discuss the results.

We have calculated the neutronic performance of coupled light water moderators in a solid beryllium reflector. The four flux-trap moderators were in the geometry depicted in Fig. 4 (except with no poisons, decouplers, and liners).
The light-water moderators were $5 \times 13 \times 13 \mathrm{~cm}$, and the solid beryllium reflector was $200 \mathrm{~cm}$ diam and $200 \mathrm{~cm}$ high. The proton energy was $800 \mathrm{MeV}$, and the light-water cooled (pure) tungsten targets were $10 \mathrm{~cm}$ diam and $22.5 \mathrm{~cm}$ long (total equivalent tungsten length).

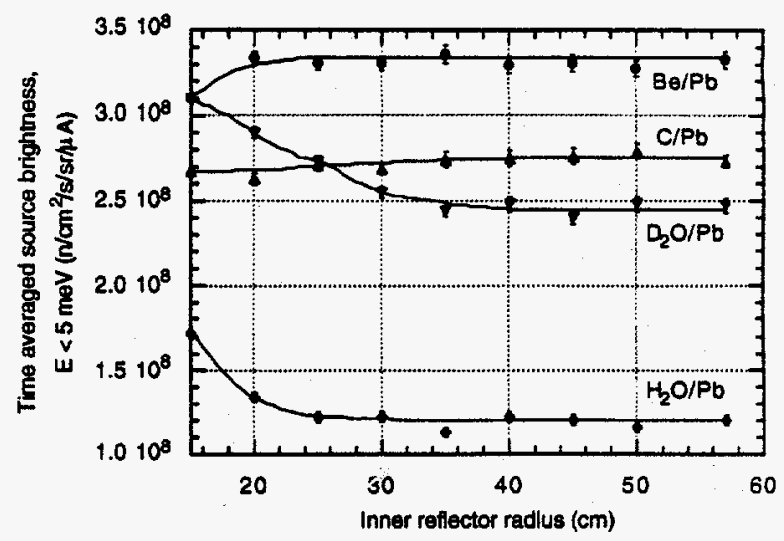

(a)

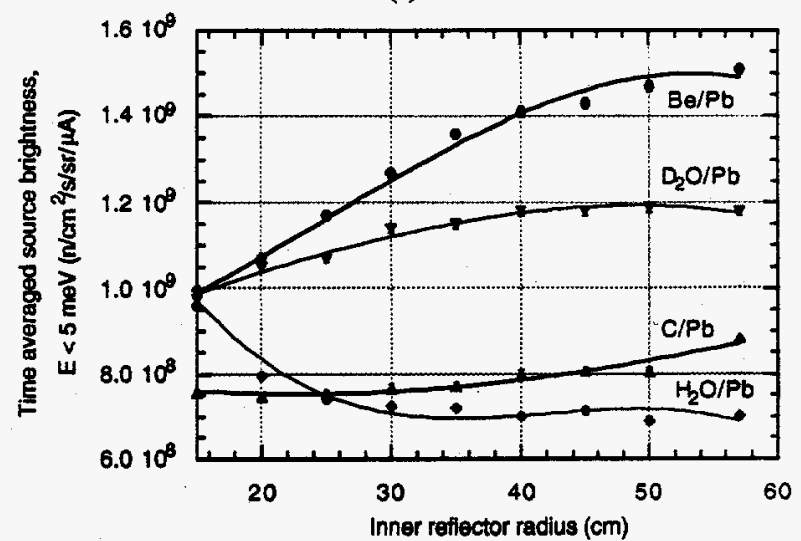

(b)

FIGURE 5. Time-averaged moderator source brightness for $5 \times 13 \times 13 \mathrm{~cm}$ liquid hydrogen flux-trap moderators (ortho/para at $50 / 50 \mathrm{v} \%$ ) for decoupled (a) and coupled (b) composite-reflector systems.

The results of this moderator thickness study are depicted in Fig. 6. Note that the time-integrated leakage flux peaks at a moderator thickness of about $4 \mathrm{~cm}$.

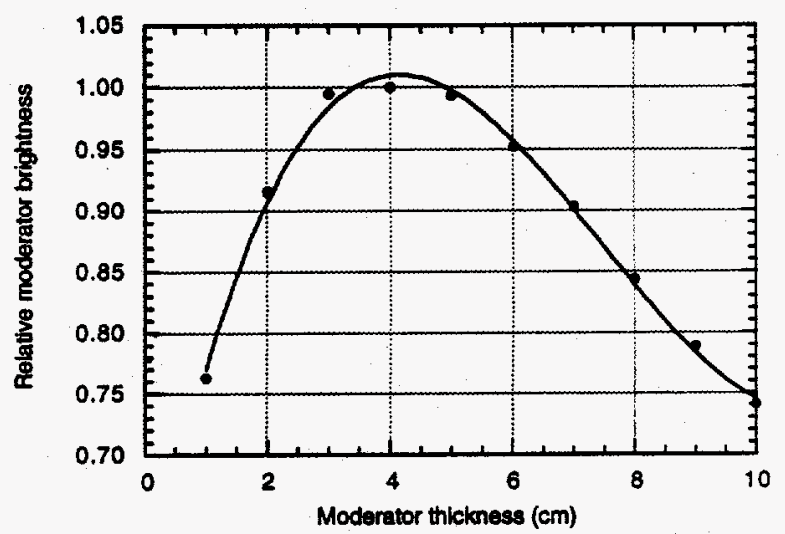

FIGURD 6. Time-averaged moderator source brightness for $13 \times 13 \mathrm{~cm}$ coupled light-water flux-trap moderators versus moderator thickness. The solid beryllium reflector was $200 \mathrm{~cm}$ diam and $200 \mathrm{~cm}$ high. 
THE MLNSC TARGET SYSTEMS

The MLNSC $800-\mathrm{MeV}$ proton beam impinges vertically downward into the target system. The innovative splittarget/flux-trap-moderator arrangement was introduced in 1983 to take advantage of the vertical proton beam injection scheme. This target system was implemented in 1985, using a composite beryllium-nickel reflector-shield(3). The split-target with four flux-trap moderators (viewed in transmission) as used in the original MLNSC as-built target system are depicted in Fig. 7a. Fig. Tb shows the upgraded MLNSC target-moderator arrangement with the addition of two upstream backscattering moderators(6). Figure 7c shows the complete MLNSC upgraded target system (targets, moderators, and reflectors) .

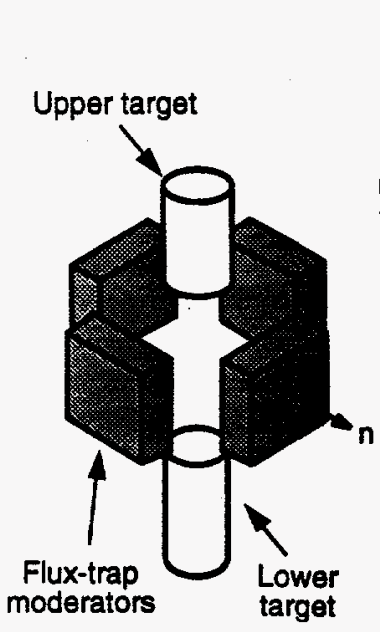

(a)

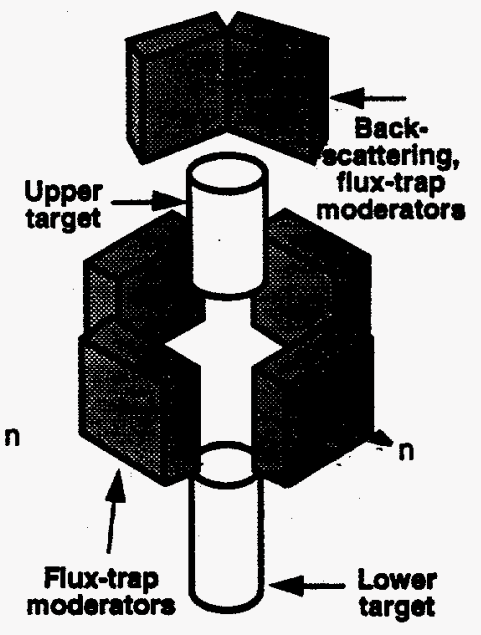

(b)

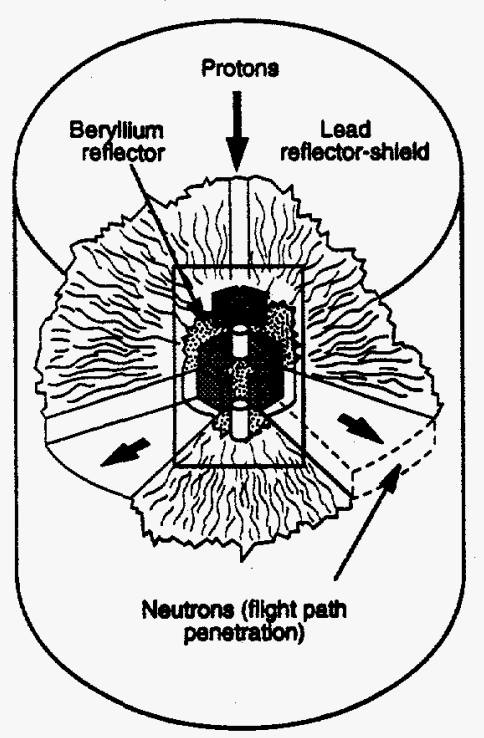

(c)

FIGURE 7. (a) the MLNSC as-built, split-target, flux-trap moderator configuration; (b) the MUNSC upgraded split-target, flux trap/backscattering moderator configuration; and (c) the MLNSC upgraded target system (targets, moderators, and reflectors).

\section{DISCUSSIONS AND CONCLUSIONS}

The upgraded MLNSC target system will employ both fluxtrap and upstream/backscattering moderators to service 16 neutron flight paths with high-intensity neutron beams for materials science research. We have calculated the relative neutronic performance of the as-built MLNSC moderators to the upgraded MLNSC moderators. One design goal was to add the two additional moderators and keep the relative performance of the four flux-trap moderators to within $10 \%$ of each other to account for engineering penalties as the design progresses. This objective has been fulfilled.

TABLE 1. Relative time averaged performance of the MLNSC Upgrade target system. The coupled $\mathrm{LH}_{2}$ is relative to the MLNSC as built decoupled $\mathrm{LH}_{2}$ and the coupled $\mathrm{H}_{2} \mathrm{O}$ is relative to the MLNSC as built $\mathrm{HI}$ $\mathrm{H}_{2} \mathrm{O}(3,4,5)$.

\begin{tabular}{|l|c|c|}
\hline & MLNSC as built & MLNSC Upgrade \\
\hline decoupled $\mathrm{LH}_{2}$ & 1.00 & 1.14 \\
\hline $\mathrm{HR} \mathrm{H}_{2} \mathrm{O}$ & 1.00 & 1.18 \\
\hline $\mathrm{HI} \mathrm{H}_{2} \mathrm{O}(3,4,5)$ & 1.00 & 1.10 \\
\hline $\mathrm{HI} \mathrm{H}_{2} \mathrm{O}(6,7,8)$ & 1.00 & 1.14 \\
\hline coupled $\mathrm{LH}_{2}$ & & 5.34 \\
\hline coupled $\mathrm{H}_{2} \mathrm{O}$ & & 6.56 \\
\hline
\end{tabular}

\section{ACKNOWLEDGMENTS}

This work was supported by the U. S. Department of Energy, BES-DMZ, under contract No. W-7405-Eng-36.

\section{REFERENCES}

1. G. J. Russell, E. J. Pitcher, and L. L. Daemen, "Introduction to Spallation Physics and Spallation-Target Design," in Proceedings of the International Conference on Accelerator-Driven Transmutation Technologies and Applications, AIP Conference Proceedings 346 (July 1994), pp. 93-104.

2. G. J. Russell, C. D. Bowman, E. R Whitaker, H. Robinson, and M. M. Meier, "LANSCE High-Power (200 uA) Target-Moderator-ReflectorShield," in ICANS-VIII: Proceedings of the Eighth Meeting of the International Collaboration on Advanced Neutron Sources, RutherfordAppleton Laboratory report RAL-85-110, pp. 272-293 (November 1985).

3. N. Bultman, A. Jason, E. Pitcher, G. Russell, W. Sommer, D. Weinacht, and R Woods, "Los Alamos Next-Generation Spallation Source," Volume I, Los Alamos National Laboratory report LA-UR-95-4300 (December 1995).

4. G. J. Russell, E. J. Pitcher, and P. D. Ferguson, "Coupled Moderator Neutronics", in ICANS-XIII: Proceedings of the 13th Meeting of the International Collaboration on Advanced Neutron Sources, G. S. Bauer and R. Bercher, Eds., Paul Scherrer Institut report PSI 95-02, ISSN 1019. 6447, pp. 495-509 (November 1995).

5. E. J. Pitcher, G. J. Russell, P. A. Seeger, and P. D. Ferguson, "Performance of Long-Pulse Source Reference Target-ModeratorReflector Configurations," in ICANS-XIII: Proceedings of the 13th Meeting of the international Collaboration on Advanced Neutron Sources, G. S. Bauer and R. Bercher, Eds., Paul Scherrer Institut report PSI 95-02, ISSN 1019-6447, pp. 323-329 (November 1995).

6. P. D. Ferguson, G. J. Russell, and E. J. Pitcher, "Reference Moderator Calculated Performance for the LANCE Upgrade Project," in ICANSXIII: Proceedings of the 13th Meeting of the International Collaboration on Advanced Neutron Sources, G. S. Bauer and R. Bercher, Eds., Paul Scherrer Institut report PSI 95-02, ISSN 1019-6447, pp. 510-517 (November 1995). 